\title{
Improving Efficiency Monitoring Systems for Potentially Dangerous Objects Based on Optimization of Group Detection Sensors
}

\author{
Boris B. Pospelov", Vladimir A. Andronov \\ National University of Civil Protection in Ukraine, Ukraine
}

Copyright (C) 2015 by authors, all rights reserved. Authors agree that this article remains permanently open access under the terms of the Creative Commons Attribution License 4.0 International License

\begin{abstract}
This paper presented the method for improving the effectiveness detecting critical states of potentially dangerous objects on the basis of the sensors group and a joint Bayesian optimization the threshold and majority rule to detection.
\end{abstract}

Keywords Monitoring System of Potentially Dangerous Objects, Group Sensor, Critical State of the Object, Detection Indexes of the Critical State

\section{Introduction}

One of the main directions to reduce potential damage from potentially hazardous industrial objects (power plants, transmission facilities, hydro engineering, oil, gas and chemical objects, pipelines, etc.) is the development of electronics systems to monitor their current state and early detection of critical states of objects. The global experience of running such systems is faced with the problem of detecting the presence of errors in the critical states of dangerous objects in the form of omissions and false alarms. Due to the continuous increase in the number of potentially dangerous industrial sites in different countries, the problem of increasing the efficiency of electronics systems monitoring is becoming particularly relevant. The priority in solving this issue should be considered as reducing errors the early detection critical conditions for hazardous industrial facilities. The most constructive in this direction is the approach based on combining several primary sources of information to the group sensors. For group sensors the presence or absence of the critical state of the object shall be made on the basis of a two-step batch processing of information from the individual sensors in a group. In this regard, the need of improving effectiveness of critical states detection by means of group sensors on the one hand, and the specific conditions of monitoring for dangerous objects, that decrease the detection efficiency on the other hand, give rise to an optimization problem for a two-stage detection of group sensors. In [1] to improve the effectiveness of fire detection is proposed unification of the primary sensors in the group and application of the majority principle of processing information from a group of sensors. At the same time reducing of wrong decisions are not considered. In [2] to reduce the erroneous decisions of the group sensor is proposed to use criterion of maximum difference between the probabilities of correct and false outcome. It is shown that for a fixed threshold for the primary sensor there is a definite rule between the number of triggered sensors and the total number of sensors, using which making decision in respect of fire is optimal in terms of the selected criteria. An equation that allows choosing the optimal rule is gotten, but is not considered the question of the threshold's choice for the primary sensors and connection with rules of majority processing.

\section{Problem Definition and its Solution}

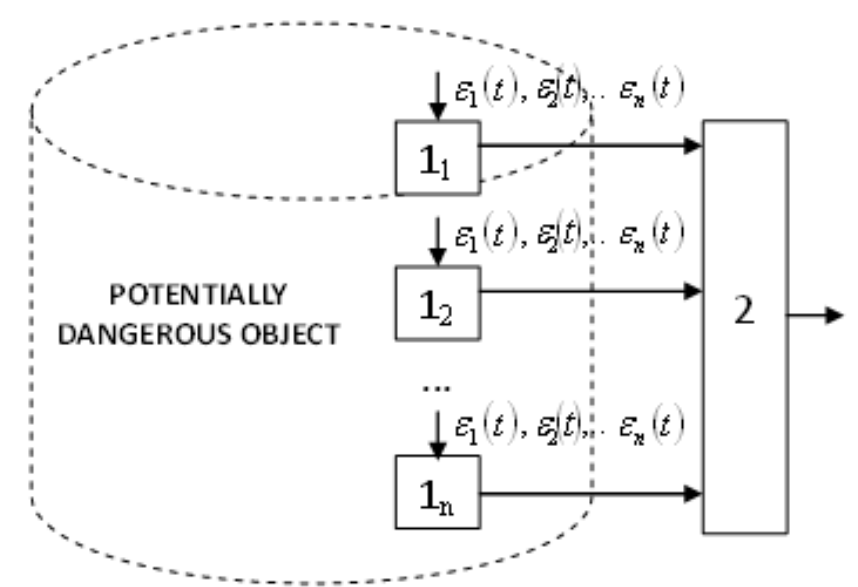

Figure 1. Structure of the group sensor: $1_{1}, 1_{2}, \ldots, 1_{n}$ - object on-site sensors, informing about the state of the object; 2 - detection device of the critical state for a dangerous object 
The aim is to increase the efficiency of electronics system detecting critical states of potentially dangerous objects of industrial field on the basis of decreasing erroneous decisions by means of the joint optimization of the threshold in primary sensors and majority rules in group sensors. We consider the group sensor, a typical structure of which is shown in Fig. 1. Object sensors generate and transmit information about of object the states, which is exposed to influence of additive random factors $\varepsilon_{1}(t), \varepsilon_{2}(t), \ldots$, $\varepsilon_{n}(t)$. In the first stage detection is carried at the sensors' level by comparing level of information signal about the critical state of object with the appropriate threshold.

The random nature of the critical state of objects, as well as confounding factors that are recorded by on-site sensors to detect the primary stage, gives rise to two types of errors: failure of the first kind - skipping in the presence of a critical state; failure of the second kind - a false alarm in the absence of a critical state. At the same time errors of the primary sensory detection will be transformed into the corresponding error detection for the group sensor. Let the error's probability of type I be the magnitude of $\alpha$ and the error's probability of type II - $\beta$ at the time of the initial detection (at the level of sensors) for a fixed threshold. Then the error's probability $p_{\alpha}$ of type I (skipping) for the group sensor, characterized by the lack of detection of the critical state for more than $n-k$ of sensors of a group in the actual presence of it, will be determined by magnitude

$$
p_{\alpha}=1-\sum_{i=k}^{n} C_{n}^{i}(1-\alpha)^{i} \alpha^{n-i}
$$

The error's probability of type II $p_{\beta}$ (false alarm), characterized by the detection of the critical state of not less than by $k$ when actually it is absent, will be determined by magnitude

$$
p_{\beta}=\sum_{i=k}^{n} C_{n}^{i} \beta^{i}(1-\beta)^{n-i} .
$$

Taking into account equations (1) and (2), in general, optimization of majority rules for detecting the critical state " $k / n "$ in the second stage of detection for the sensor in the group should be carried out in accordance with the Bayesian criterion, which determines the average risk of erroneous solutions

$$
\begin{aligned}
L & =A p_{\alpha}+B p_{\beta} \\
& =A-\sum_{i=k}^{n} C_{n}^{i}\left[A(1-\alpha)^{i} \alpha^{n-i}-B \beta^{i}(1-\beta)^{n-i}\right] \rightarrow \min _{k}
\end{aligned}
$$

where $A, B$ - generalized weighting coefficients: $A \geq 0, B \geq 0$. Coefficients $A, B$ can be determined by the probabilities that are related to errors of various events, as well as by the value of damage or damage of errors and multiplication of damage and probability of the associated events. The problem of minimizing the Bayesian risk (3) is equivalent to maximizing

$$
\sum_{i=k}^{n} C_{n}^{i}\left[A(1-\alpha)^{i} \alpha^{n-i}-B \beta^{i}(1-\beta)^{n-i}\right] \rightarrow \max _{k}
$$

Let the $1-\alpha>\beta$. Following [2], the maximum of equation (4) can be reached at a value $k$ equal to the top of the nearest $x_{0}$ integer, but not larger than $n$. The desired value $x_{0}$ is given by

$$
x_{0}=\frac{\ln \frac{B}{A}+n \ln \frac{1-\beta}{\alpha}}{\ln \left(\frac{1-\alpha}{\beta} \cdot \frac{1-\beta}{\alpha}\right)} .
$$

At the expression (5) can be seen, that the choice of the $k$ sensors detected a critical state of the object necessary for optimal solutions in the sensor group in the sense of minimizing the Bays' risk (3), it depends on the probability of errors in the single sensor and generalized $A$ and $B$ weighting factors. Methods of assessing the effect of the weighting factors on the value of the optimum number of single sensors for fixed values $\alpha$ and $\beta$ of the probability of errors of individual sensors is dedicated to the work [3]. It should be noted, that the probability $\alpha$ and $\beta$ of error sensors essentially depend on the statistics of the critical state of a dangerous object, hindering factors and the selected threshold. In this regard, important for applications is the problem of the joint selection of sensory thresholds and majority rules for the detection of the critical state of the sensor group. Let the statistics of the observed states $T=T p+\varepsilon$ of the dangerous object in the event $T p$ of a critical state and the random factors $\varepsilon$ is determined by the density $\mathrm{P}_{1}(\mathrm{~T})=\frac{1}{\sqrt{2 \pi} \sigma} \mathrm{e}^{-\frac{(\mathrm{T}-\mathrm{Tp})^{2}}{2 \sigma^{2}}}$, and $\mathrm{P}_{0}(\mathrm{~T})=\frac{1}{\sqrt{2 \pi} \sigma} \mathrm{e}^{-\frac{\mathrm{T}^{2}}{2 \sigma^{2}}}$ in his absences. Then the probability $\alpha$ of missing and $\beta$ false detection for sensors for the given threshold $\mathrm{u}$ will be determined accordingly:

$$
\begin{array}{r}
\alpha(u)=\int_{-\infty}^{u} \frac{1}{\sqrt{2 \pi} \sigma} e^{-\frac{(T-T p)^{2}}{2 \sigma^{2}}} d T, \\
\beta(u)=\int_{u}^{\infty} \frac{1}{\sqrt{2 \pi} \sigma} e^{-\frac{T^{2}}{2 \sigma^{2}}} d T .
\end{array}
$$

In view of (6), the average risk of the Bayesian detection sensor group will be determined by functionality

$$
L(k, n, u)=A p_{\alpha}(k, n, u)+B p_{\beta}(k, n, u) \rightarrow \min _{n, k, u},
$$




$$
\begin{gathered}
p_{\alpha}(k, n, u)=1-\sum_{i=k}^{n} C_{n}^{i}(1-\alpha(u))^{i} \alpha(u)^{n-i}, \\
p_{\beta}(k, n, u)=\sum_{i=k}^{n} C_{n}^{i} \beta^{i}(u)(1-\beta(u))^{n-i} .
\end{gathered}
$$

In general, the optimization of the sensor group in accordance with a Bayesian criterion (7) must be performed together the number $n$ and the threshold $u$ of sensors in the group, and the number $k$ of sensors, which detect a critical state of the object. In practice, the total number $n$ of sensors in the group is normally fixed. Therefore it is the joint optimization of the value $k$ and the threshold $u$ sensors. The threshold $u$ is determined by current the statistical properties of the critical factors and interference Therefore, the solution of the optimization problem for the sensor group in the general case depends on the statistics of observations the critical states.

For example, key indicators of detection quality for considered test conditions for sensors group, with optimized only the number $k$, and for sensors group with jointly optimized number $k$ and thresholds $u=a$, for total number $n$ of sensors in a group is equal to 20 are presented in Fig. 2 a, b. On the Fig. 2 a, b the value $U o$ and $\mathrm{k}_{\mathrm{O}}$ determined for a joint optimization $k$ and the threshold $a=u$ for the sensors. And through $D(\cdot)$ and $L T(\cdot)$ are denoted functional for the probabilities of the correct detection of a critical state and a false alarm for group sensors. In Fig. 2 submitted for comparison the values of the probabilities of correct detection $D(T p)$ and false alarm $\beta(T p)$ for a single sensor and dependence of the average risk for the groups sensor $R 5(\cdot)$ with optimized threshold $U o$ and for the groups sensor $L 5(\cdot)$ with a fixed threshold $T p$ and corresponding $D(T p)$ and $\beta(T p)$.

Analyzing dependencies in Fig. 2 it is evident that the quality factors of detection in group sensors that is optimized by the value $k$ and threshold $u=a$ for sensors substantially increase with increasing ratio $T p / \sigma$. For example, the probabilities of correct detection and false alarm for optimized by two parameters for ratio $T p / \sigma=1$ are respectively 0,954 and 0,026 . Similar characteristics of detection for a group sensor that is optimized only by the number $k$ are respectively 0.942 and 0.029 and for a single sensor $-0,5$ and 0,159 . For the ratio $T p / \sigma=2$ of the probability of correct detection and false alarm for group sensor that is optimized by two parameters are 0,999 and $2,504 \cdot 10^{-5}$ respectively, and for the group sensor that is optimized only by value $k-0,999$ and $9,691 \cdot 10^{-4}$ respectively. Represented data indicate that in the case of sensors group, which is optimized jointly by $k$ and threshold $u=a$, it is possible lower the value for probability of false alarm with the same probability of correct detection equal to 0,999 . At the same time increase of value the ratio $T p / \sigma$ leads to an even greater gain in these considered indicators detection of critical states of the objects.

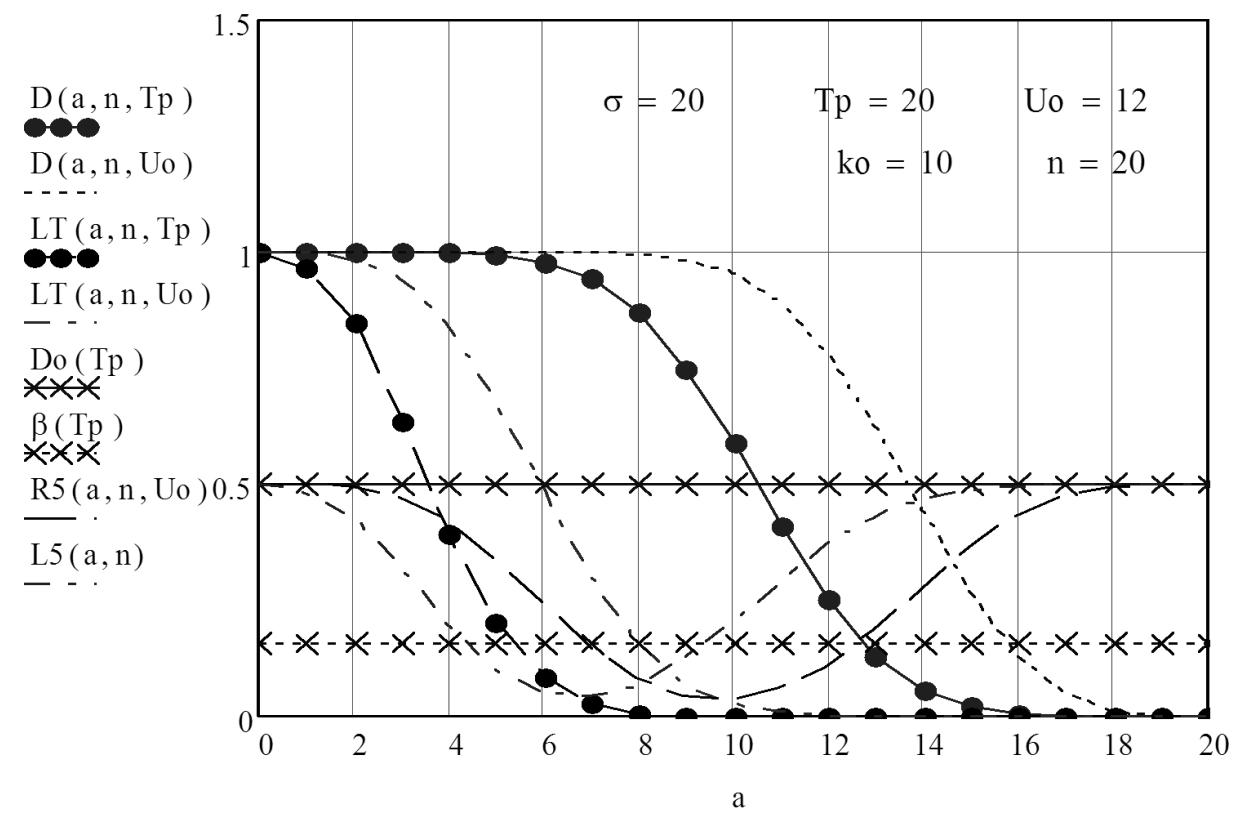




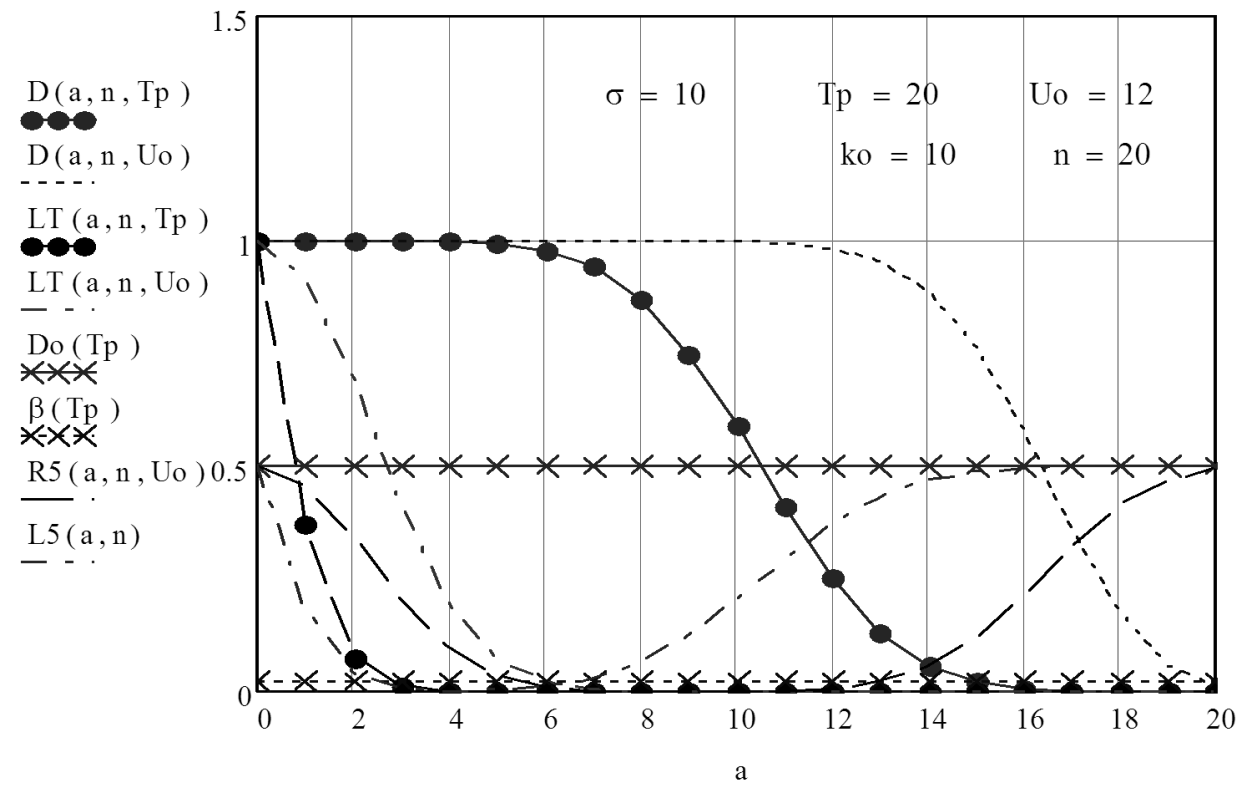

b)

Figure 2. Characteristics of group sensor's detection for different conditions of observation

\section{Conclusions}

This paper is presented the method for improving the effectiveness detecting critical states of potentially dangerous objects on the basis of the sensors group and a joint Bayesian optimization the threshold and majority rule to detection. The simulation results reveal that the threshold and majority rule to detection are not independent. This dependence has a mathematically complex representation (7). The dependence of the threshold and the detection rule is investigated in various conditions of observation. It is shown that for improve efficiency detection on the presented method the thresholds value and majority rule they must be optimized together. Quantitative assessment of expected improve detection for joint optimization of the thresholds and majority rules indicates high efficiency of the presented method for group sensors.

\section{REFERENCES}

[1] Maintenance of fire alarm system. Fire Safety Eng. - 2003. -10. N.5, pp. 3-5.

[2] Pospelov B.B., Basmanov A.E., Mikhailuk A. A., Kulik Y. S. Optimal choice for number of fire detectors in the protection of oil reservoir. Problems of Fire Safety. - H.: NUGZU, 2011. - Vol. 30, pp. 12-15.

[3] Pospelov B. B., Basmanov A. E. The structural method of increasing reliability of the sensors of the primary information in the system to reduce impact of emergency. Problems of urgency situations. - H.: NUGZU, 2011. - Vol. 14, pp. 129-134. 